\title{
Didactic proposal for teaching the geometrical optics with high school students
}

D. C. Ramirez-Moyano, F. A. Monroy-Ramirez

D. C. Ramirez-Moyano, F. A. Monroy-Ramirez, "Didactic proposal for teaching the geometrical optics with high school students," Proc. SPIE 9793, Education and Training in Optics and Photonics: ETOP 2015, 979334 (8 October 2015); doi: $10.1117 / 12.2223256$

SPIE Event: Education and Training in Optics and Photonics: ETOP 2015, 2015, Bordeaux, France 


\title{
DIDACTIC PROPOSAL FOR TEACHING THE GEOMETRICAL OPTICS WITH HIGH SCHOOL STUDENTS
}

\author{
D.C. Ramirez-Moyano ${ }^{\mathrm{a}}$, F.A. Monroy-Ramirez ${ }^{\mathrm{a}, \mathrm{b}^{*}}$ \\ ${ }^{a}$ Science Education Area, National University of Colombia, Bogota, Colombia \\ ${ }^{\mathrm{b}}$ Applied Optics Group, Physics Department, National University of Colombia, Bogotá, \\ Colombia, famonroyr@unal.edu.co
}

\begin{abstract}
This paper presents a methodological approach to the teaching of geometrical optics based on a review and preliminary analysis of the concepts of elementary geometry. The previous review of geometry elements necessary and sufficient for the construction of the images given by reflection and refraction at plane surfaces, allows the students the interpretation, representation and description of these images with a higher level of conceptualization of physical phenomena, the levels obtained by the conventional ways of teaching, because the student focuses his attention on the physical phenomenon, without worrying at the same time to understand the geometrical tool. The methodological approach was performed with thirty students from a basic secondary school in Bogota-Colombia and, based on some geometry workshops, the students acquired the foundations of geometry giving meaning to geometric constructions of phenomena of reflection and refraction at plane surfaces and subsequently the development of workshops on tracing rays and some laboratory practices, the phenomena of geometrical optics was expanded. This was carried out using the Active Learning Methodology and Solving Problem Methodology. The efficiency of the proposal was measured by comparing the results of a diagnostic test and an output test, where the progress by the students was demonstrated.
\end{abstract}

Keywords: geometrical optics, concepts of elementary geometry, Active Learning Methodology, Solving Problem Methodology, didactical proposal.

\section{INTRODUCTION}

Geometrical optics is a model that represents and analyzes the reflection phenomena, refraction and dispersion of light in the macroscopic appearance, using geometric constructions which underlie comparisons established between the object and the image forming system, which may be a mirror, a lens or a combination thereof [1].

However, in the teaching and learning process of these concepts specifically in the subject of physics, it has become evident in the eleventh grade students, some shortcomings in regard to the understanding of concepts associated with the phenomena of light reflection and refraction, in the interaction with different media, as well as in the interpretation of the image formation and their description. The difficulties listed above come from ignorance of basic elementary geometry concepts and procedures. Although those are issues that are part of the curriculum of secondary basic education in Colombia, when used in the geometric construction of the observed phenomena, they lack meaning for students.

Given that geometry is a "tool to interpret, understand and appreciate a world that is predominantly geometric" [2] it emerges the interest of strengthening some concepts and relationships of elementary geometry in students, necessary for the understanding of representations of phenomena associated with geometrical optics. For this purpose, it was proposed the following question which guided the development of the didactic proposal of this paper: What features should a teaching strategy have, that could allow a ninth grade student in Colombia to interpret, understand and describe the images obtained from reflection and refraction, according to the geometrical optics model?

This work is based on a review and analysis of the geometry elements that are the starting point used by the geometrical optics model, which supports the construction of geometric representations of the reflection and refraction of light on plane surfaces.

Education and Training in Optics and Photonics: ETOP 2015, edited by Eric Cormier, Laurent Sarger Proc. of SPIE Vol. 9793, 979334 · @ 2015 SPIE, IEEE, OSA, ICO · doi: 10.1117/12.2223256 
The strategy was made with 30 ninth grade students between 14 and 16 years of age from "Gimnasio Mixto Manuel del Socorro Rodríguez school- (GMMSR)” located in Suba (Bogota-Colombia). The Institutional Education Project (IEP) is based on values education and a business emphasis.

A didactic proposal was performed applied and validated with two simultaneous approaches. First, it was developed geometry and geometric optics workshops aimed to deepen the images construction teaching by means of ray tracing, emphasizing on the phenomena of reflection and refraction when light falls on flat surfaces. In the second part, experimental practices with elements of low cost and easy to obtain were implemented, which allowed the assimilation and internalization of light involved macroscopic phenomena by the students.

The teaching strategy used is based mainly on the Methodology of Active Learning [3], Problem Solving and geometric constructions were performed using Geogebra dynamic software.

\section{IMPLEMENTATION AND VALIDATION OF DIDACTIC PROPOSAL}

Implementation and validation of didactic proposal was based on its application with the group of students in four different stages: (1) Input test (2) Implementation of theoretical workshops and experimental work practices, (3) Final test (4) Results analysis.

At the beginning of the process an input test was conducted to explore the background of the students on the elementary geometry basis, specifically in terms of lines, angle measurements, congruence and similarity of triangles [4] as well as on elementary geometric optics, specifically imaging by reflection and refraction on flat surfaces.

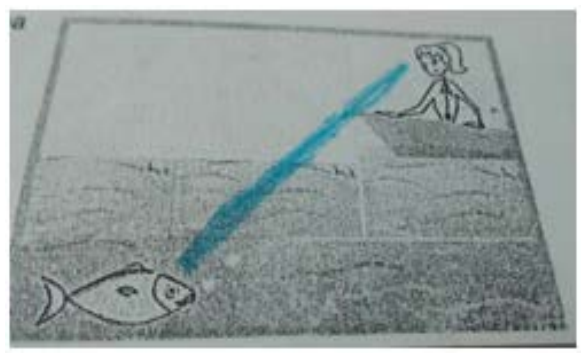

(a)

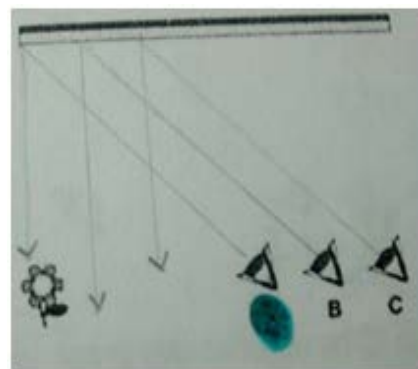

(b)

Figure 1: Drawings made by students in the entrance test showing the path of light in (a) refraction and (b) reflection of light.

According to the diagnostic activity results, the difficulties for students to establish relationships between the concepts of geometry and geometric representations of the phenomena of reflection and refraction of light were evident, specifically recognizing the beam path of light, both reflection and refraction. And the difficulty was also identified in the process of determining similarity relations and consistency of triangles in modeling situations phenomena of light reflection and refraction.

As for the workshops, two workshops were conducted in terms of elementary geometry: in order to identify the concepts associated with elementary geometry and relationships that can be established between its elements there were two pilot workshops for each topic. Two pilot workshops on light reflection and refraction, where basic concepts of elementary geometry to the phenomena of reflection and refraction of light were applied, and two geometrical optics workshops related to the construction of images from light reflection and refraction, depending on the model of geometrical optics. 


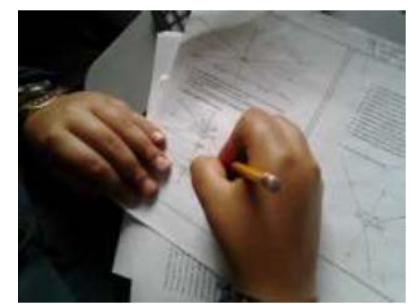

(a)

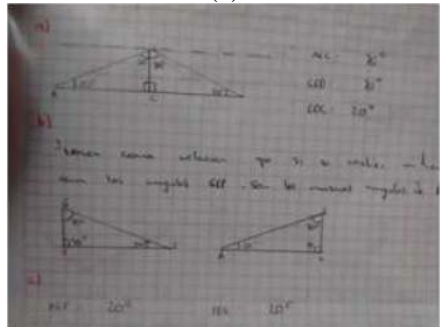

(c)

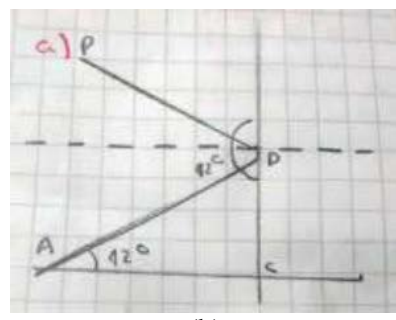

(b)

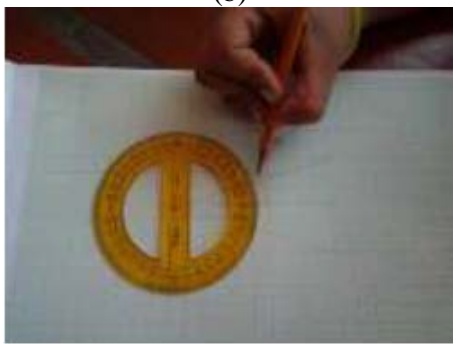

(d)

Figure 2: Drawings made by students in theoretical geometry workshops in order to identify the elements necessary for the construction of a reflection given by image.

Workshops on plane geometry gave students the opportunity to identify and assimilate the applicable fundamentals for the construction of images by ray tracing reflection and refraction.

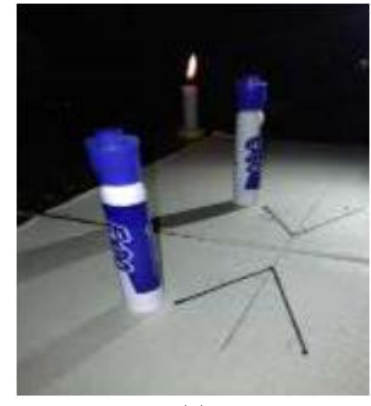

(a)

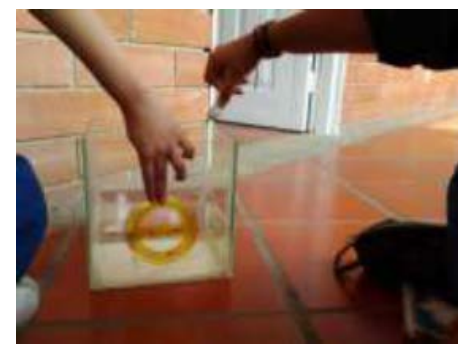

(c)

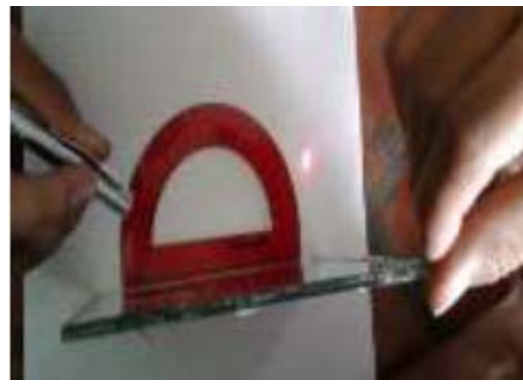

(b)

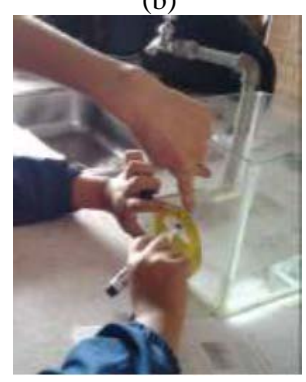

(d)

Figure 3: Experimental workshops with students about reflection of light (a) and (b); light refraction (c) and (d)

Experimental workshops for students allowed them to assimilate and understand the implicit geometric elements in the description of the light reflection and refraction trajectory.

The output test was performed exactly as the input test in order to have the option to compare the results. This test showed that through the implementation of the proposal, students successfully solved the situations which explored concepts of basic geometry and relationships between geometric constructions, as well as characterizing phenomena of light reflection and refraction in reference to its trajectory, the object and the image and the incidence, reflection, refraction and deviation angles involved. 


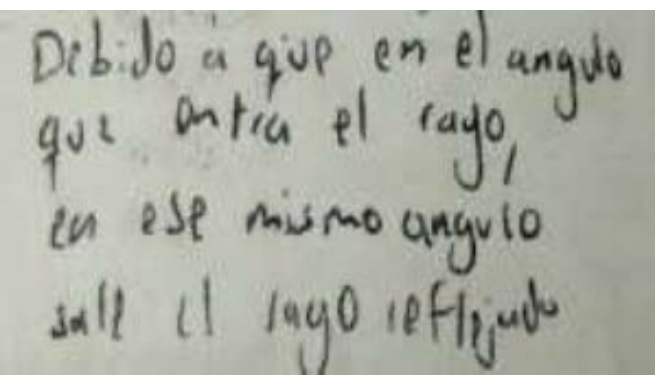

(a)

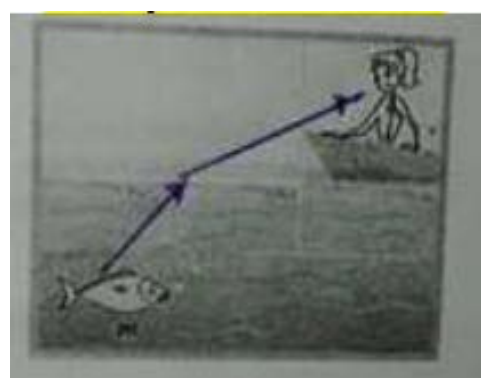

(b)

Figure 4: Answers in the final test (a) Answer to description of the light reflection and (b) Drawing by students describing the refraction of light

\section{DESCRIPTION AND RESULTS ANALYSIS}

The comparison between the results obtained from the input and output test allowed to have an idea of the success of the implementation of a methodological approach to this group of students.

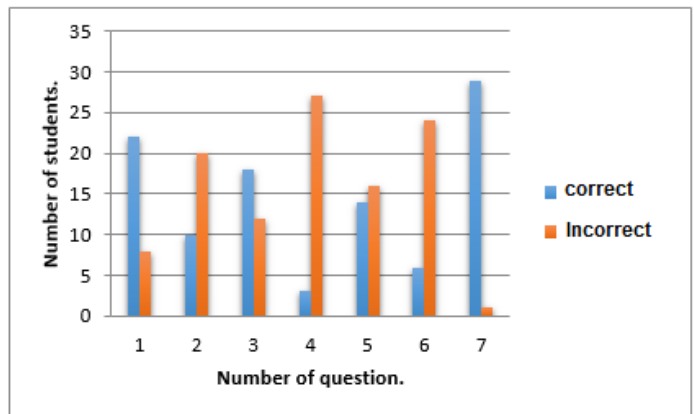

(a)

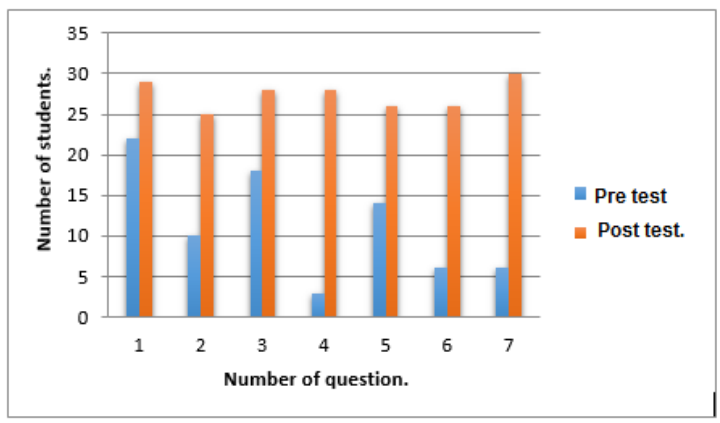

(c)

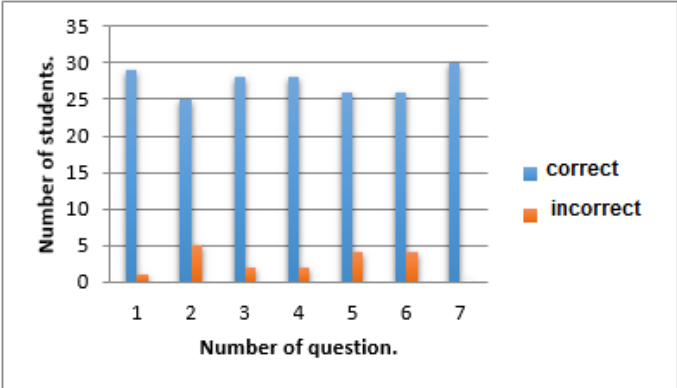

(b)

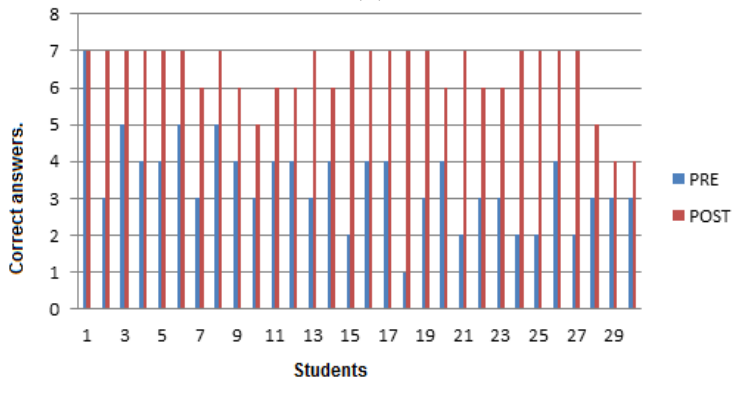

(d)

Figure 5: (a) Results for input test; (b) Results for output test; (c) Contrast between the input test and the output test; (d) Number of correct answers per student in input and output test

Figure 5 (a) shows the results for all students in the input test. It is prevailing the high percentage of wrong answers, which shows the students initial conceptual deficiencies at the beginning of the teaching and learning process. Figure 5 (b) shows the results for all students in the final test, it is clearly evident the change between (a) and (b) with the number of correct answers. When comparing the results (Figure 5-c), it shows the number of correct answers obtained by students on each tests, supporting the progress of the students as a result of the teaching-learning process performed both theoretically and experimentally. The same result can be evidenced in Figure 5 (d) in which the heights of the red bars representing the correct answers in the test are predominant. 
To analyze the results above in quantitative terms, the conceptual gain in the proposal implementation can be measured by confronting the results between the initial test and the final test. Afterwards, the data is valued with the normalized gain given by Hake [5] which provides the ability to measure and compare the conceptual gain results from the normalized gain $<\mathrm{g}>$, which is defined as the increase ratio in input test and final test in respect to the highest possible value:

$$
<g>=\frac{\%<S f>-\%<S i>}{100-\%<S i>}
$$

Where $\%<S f>$ is the percentage obtained in the output test and $\%<S i>$ is the percentage obtained in the input test. Considering the data presented in Table 1, the Hake gain is calculated for each student using equation (1) which is shown in the fourth column of the same table.

\begin{tabular}{|c|c|c|c|}
\hline STUDENT & \%INPUT & \%OUTPUT & G.Hake \\
\hline 1 & 100 & 100 & 0 \\
\hline 2 & 40 & 100 & 1 \\
\hline 3 & 70 & 100 & 1 \\
\hline 4 & 56 & 100 & 1 \\
\hline 5 & 56 & 100 & 1 \\
\hline 6 & 70 & 85 & 0,5 \\
\hline 7 & 40 & 100 & 1 \\
\hline 8 & 70 & 85 & 0,5 \\
\hline 9 & 56 & 70 & 0,32 \\
\hline 10 & 40 & 85 & 0,75 \\
\hline 11 & 56 & 85 & 0,66 \\
\hline 12 & 56 & 100 & 1 \\
\hline 13 & 40 & 100 & 1 \\
\hline 14 & 56 & 85 & 0,66 \\
\hline 15 & 30 & 100 & 1 \\
\hline 16 & 56 & 100 & 1 \\
\hline 17 & 56 & 100 & 1 \\
\hline 18 & 15 & 100 & 1 \\
\hline 19 & 40 & 100 & 1 \\
\hline 20 & 56 & 85 & 0,66 \\
\hline 21 & 30 & 100 & 1 \\
\hline 22 & 40 & 85 & 0,75 \\
\hline 23 & 40 & 85 & 0,75 \\
\hline 24 & 30 & 100 & 1 \\
\hline 25 & 30 & 100 & 1 \\
\hline 26 & 56 & 100 & 1 \\
\hline 27 & 30 & 100 & 1 \\
\hline 28 & 40 & 70 & 0,5 \\
\hline 29 & 40 & 56 & 0,27 \\
\hline 30 & 40 & 56 & 0,27 \\
\hline
\end{tabular}

Table 1. Registration percentage obtained by each student.

To set the group gain in respect to the results of the diagnostic activity and the output test, equation (2) was used, which calculates the average normalized gain $\langle\overline{\mathrm{g}}\rangle$ from the average of the normalized gain with the group of 30 students.

$$
<\overline{\mathrm{g}}>=\frac{1}{\mathrm{n}} \sum_{\mathrm{i}=1}^{\mathrm{n}} \mathrm{gi}
$$

Where $\mathbf{n}$ is the number of students who presented the input and output test and $<\overline{\mathrm{g}}>$ the gain represented in Table 1. 
Replacing the data in column 4 -Table 1 , the average normalized gain group value is obtained to give $<\overline{\mathrm{g}}>=$ 0,77 .

The normalized gain obtained in equation (2) can categorize the data into three areas: low, medium and highnormalized gain as shown below:

\begin{tabular}{|c|c|}
\hline Zone & Interval \\
\hline Low & $g<0,3$ \\
\hline Medium & $0,3<g<0,7$ \\
\hline high & $g>0,7$ \\
\hline
\end{tabular}

Table 2. Areas of normalized gain.

According to Table 2 and given the result in equation (2) $<\bar{g}>=0,77$ normalized gain group can be categorized as high; which verifies the results shown in the tables and the analysis done. Based on the above, the effectiveness of teaching proposal is confirmed, according to the progress showed in test results and the increasing understanding gained by the students related to the characteristics of the phenomena and their representation using geometric models.

\section{CONCLUSIONS}

From the analysis of the results based on the gain Hake for each of the students, it was shown that about $60 \%$ of them were located in an area high standard gain, while 30\% were located in an average gain area and $10 \%$ in a low-gain area, which let us conclude that the proposal was accurately used effectively at the individual level.

At group level, for the group it was obtained an average normalized gain $<\overline{\mathrm{g}}>=0,77$, according to Hake, it lies within an area of high normalized gain, which clearly indicates that the results of the proposal were effective in relation to the fact of establishing solid geometric bases that are part of the representations used to model the phenomena of light reflection and refraction.

\section{REFERENCES}

[1] E. Hecht, Optics, 4th Ed., Addison Wesley, (2002).

[2] Ministry of National Education curriculum guidelines for the area of mathematics. Bogotá, Colombia (1998).

[3] D. Sokoloff ALOP Training Manual, "Active Learning in Optics and Photonics", pp (22 to 26), (2006).

[4] O. J. Cardona, Basic Geometry. Universidad Pontificia Bolivariana, Medellin, Colombia, (2004).

[5] R. Hake, Am J. Phys 66, 64. Interactive-engagement vs. traditional methods: A six-thousand-student survey of mechanics test data for introductory physics courses, (1998). 